\title{
Stefano G. Caneva (ed.), The Materiality of Hellenistic Ruler Cults
}

Liège, Presses Universitaires de Liège (Kernos. Supplément 35), 2020, pp. 300, ISBN 978-2-87562-242-6, € 30

\section{Claudio Biagetti}

\section{(2) OpenEdition}

\section{Journals}

\section{Edizione digitale}

URL: https://journals.openedition.org/mythos/3872

DOI: $10.4000 /$ mythos.3872

ISSN: 2037-7746

Editore

Salvatore Sciascia Editore

Notizia bibliografica digitale

Claudio Biagetti, «Stefano G. Caneva (ed.), The Materiality of Hellenistic Ruler Cults», Mythos [Online],

15 | 2021, online dal 15 décembre 2021, consultato il 15 décembre 2021. URL: http://

journals.openedition.org/mythos/3872 ; DOI: https://doi.org/10.4000/mythos.3872

Questo documento è stato generato automaticamente il 15 décembre 2021.

Mythos 


\section{Stefano G. Caneva (ed.), The Materiality of Hellenistic Ruler Cults}

Liège, Presses Universitaires de Liège (Kernos. Supplément 35), 2020, pp. 300, ISBN 978-2-87562-242-6, € 30

\section{Claudio Biagetti}

\section{NOTIZIA}

Stefano G. Caneva (ed.), The Materiality of Hellenistic Ruler Cults Liège, Presses Universitaires de Liège (Kernos. Supplément 35), pp. 300, ISBN: 978-2-87562-242-6, $30 €$

1 Prodotto del programma di ricerca dal titolo Practicalities of Hellenistic Rulers Cult (PHRC) (Università di Padova - Université de Liège, 2015-2020), il volume curato da Stefano G. Caneva, con la collaborazione di Luca Lorenzon, raccoglie gli atti delle giornate di studio tenute presso l'Università di Liegi (31 maggio - 1 giugno 2018), dedicate all'approfondimento degli aspetti rituali, pratici, organizzativi e - in una parola - più squisitamente materiali della celebrazione del culto in onore dei sovrani ellenistici. I saggi, ripartiti tematicamente in tre sezioni (Media, Supports, and Circulation; Ritual Space and Practice; Agency, Administration, and Funding), sono preceduti da un'introduzione del Curatore (Introduction. Rituals, Materiality, and the Cultic Honours for Hellenistic Political Leaders, pp. 9-18), il quale illustra la genesi del progetto, gli approcci e la metodologia adottati nell'esame della documentazione, gli obiettivi della ricerca. Oltre alla rielaborazione degli interventi tenuti in occasione dell'incontro di Liegi, il volume è arricchito da due ulteriori contributi, l'uno dovuto allo stesso Caneva, l'altro frutto della collaborazione di questi con Lorenzon.

2 Il saggio di Caneva che dà avvio al volume esemplifica al meglio le potenzialità euristiche di un'indagine sulla materialità dei supporti che recano testimonianza del culto reale («L'importance de la matérialité. Le rôle des petits autels, plaques et bases inscrits dans la compréhension des cultes pour les souverains», pp. 21-64): si tratta di 
uno studio su monumenti iscritti di piccole dimensioni articolato in due sezioni, ciascuna delle quali si concentra sull'esame dei contenuti, delle funzioni e dei contesti di dedica di testi celebranti, rispettivamente, Arsinoe II e i re pergameni Attalo I ed Eumene II. La sintassi del testo e le caratteristiche paleografiche, la tipologia del supporto e il materiale con cui esso è realizzato, il contesto archeologico di ritrovamento e - se diverso - quello di origine del manufatto offrono, considerati singolarmente o nel loro complesso, indicazioni fondamentali a proposito dello status dei dedicanti e delle ragioni stesse della dedica.

3 Il lavoro di Olga Palagia prende in esame l'iconografia statuaria dei Tolomei e degli Attalidi, operando una distinzione fra rappresentazioni a scopo di culto e altro tipo di immagini celebrative («The cult statues of the Ptolemies and the Attalids», pp. 65-81). Elemento discriminante nell'individuazione di una statua di culto risulta essere, nell'indagine della Palagia, il materiale con il quale l'opera venne realizzata, giacché gli

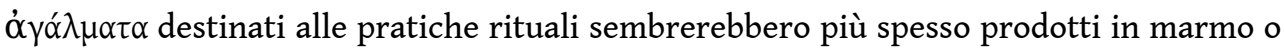
nella tecnica mista dell'acrolito. La rappresentazione dei sovrani con attributi iconografici tipici di divinità egiziane o olimpiche, inoltre, concorre all'identificazione di una statua di culto, riconoscendo de facto nella figura del sovrano - sia esso vivente o defunto - l'immagine di un dio.

4 Dedicato al formulario rituale delle cerimonie sacrificali è lo studio di Stefan Pfeiffer («Offerings and Libations for the King and the Question of Ruler-Cult in Egyptian Temples», pp. 83-102), il quale si diffonde nell'analisi del sintagma úđźp + gen. (o «hyper-formula») in testimonianze provenienti dall'Egitto tolemaico, tentando di precisarne il significato e la funzione. Presente in numerosi testi di dedica nella forma

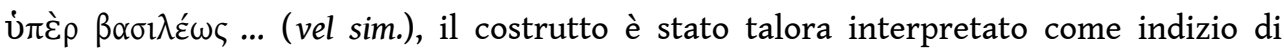
un'associazione del culto reale al culto divino, nonché come indicazione di un'offerta presentata ad una divinità a nome del sovrano («by proxy of the king»). Al termine di un'articolata disamina, tuttavia, Pfeiffer opta per una diversa spiegazione del sintagma che, sulla scorta di un consolidato uso rituale risalente al formulario già in voga nell'Egitto faraonico, farebbe piuttosto appello alla divinità al fine di preservare la buona salute del sovrano ("for the well-being of the king»).

5 Il contributo 'a quattro mani' di Rolf Strootman e Christina G. Williamson, incentrato sulla trasformazione e sulla manipolazione dello spazio urbano e suburbano nella Caria ecatomnide («Creating a Royal Landscape: Hekatomnid Use of Urban and Rural Sacred Sites in Fourth-Century Karia», pp. 105-124), può apparire a prima vista eccentrico rispetto alle tematiche ellenistiche considerate nel volume. Tuttavia, come illustrano opportunamente i due autori, l'esperienza ecatomnide ben prefigura la nuova concezione dello spazio di Alessandro e dei Diadochi, ideologicamente vòlta all'esaltazione del sovrano e alla celebrazione del culto reale. A questo riguardo, il complesso di Labraunda e la polis di Alicarnasso rappresentano casi di studio esemplari nei quali è possibile osservare come l'ambizioso programma architettonico-urbanistico degli Ecatomnidi e il sapiente sfruttamento del paesaggio naturale raggiungano effetti di spettacolarizzazione tali da conferire visibilità monumentale alla dinastia regnante, avvicinando la figura stessa del sovrano a quella di divinità come Zeus o Apollo.

6 Orientando nuovamente lo sguardo sulla dimensione privata (o, si potrebbe dire, semiprivata), lo studio di Mario C.D. Paganini si concentra sulla celebrazione del culto reale nei ginnasi e nelle associazioni della chora egiziana ( $«$ Cults for the Rulers in Private Settings: The Gymnasia and Associations of Hellenistic Egypt», pp. 125-145). La scelta di 
accomunare nell'indagine due entità apparentemente diverse come ginnasi e associazioni risiede essenzialmente negli aspetti di analogia della loro articolazione interna, della loro gestione finanziaria e amministrativa, delle loro attività culturali. Benché la critica moderna abbia considerato i ginnasi come la sede - per così dire - più naturale per la celebrazione del culto reale in Egitto, la documentazione superstite, a dire il vero assai ristretta per numero e qualità dell'informazione, non sembra però presentare un carattere così univoco, al punto che, almeno sino al momento attuale, si può disporre appena di una o due testimonianze esplicite in proposito. Diversamente, $\mathrm{i}$ regolamenti di associazioni a carattere privato trasmettono pressoché sistematicamente prescrizioni sulle modalità di celebrazioni di sacrifici in onore del sovrano, il che induce a ritenere che, effettivamente, il culto reale rappresentava un aspetto di primo piano nella vita di tali circoli, consentendo ai suoi membri, singolarmente e collettivamente, di mostrarsi leali al potere monarchico e di apparire rispettabili in seno alla società.

7 Facendo seguito ad un precedente intervento apparso nel 2018 in Epigraphica Anatolica, il secondo contributo di Caneva in questo volume torna a prendere in esame i contenuti di I.Pergamon I 246, documento che registra la concessione di onori ad Attalo III per la felice conclusione di una campagna militare («Les honneurs cultuels pour Attale III à Pergame [IvP I 246]», pp. 147-164). In questa sede, l'Autore rivolge la propria attenzione alle due fasi nelle quali dovette articolarsi l'attribuzione di onori al sovrano: l'una, sancita da una prima risoluzione da parte della comunità pergamena, prevedeva una cerimonia di accoglienza del sovrano vittorioso (apantesis), le cui procedure erano preventivamente stabilite sin nei minimi dettagli; l'altra, scandita da un'ulteriore risoluzione, doveva seguire al rientro di Attalo in città e culminare con l'attribuzione di onori di più lunga durata, fra i quali anche la condivisione del santuario di Asclepio e dell'altare di Zeus nell'agorà. Come lascia emergere la disamina di Caneva, a questi due luoghi di culto il popolo pergameno associava - si direbbe, tradizionalmente - la dimensione militare e la dimensione civile dell'operato del sovrano, ivi celebrato in rapporto di 'gerarchia variabile' con la divinità dedicataria.

8 Catharine C. Lorber propone una rassegna di documenti papiracei e, in misura minore, epigrafici che ben illuminano le pratiche di finanziamento del culto reale tolemaico («Who Pays the Bill? Monetary Aspects of Royal Cult in the Ptolemaic Kingdom», pp. 167-193). Promosse e sostenute dagli stessi Tolemei, le forme di devozione nei confronti dei membri della casa regnate furono rese possibili non soltanto attraverso $i$ fondi messi direttamente a disposizione dalle casse reali, ma anche grazie ai proventi di imposte ad hoc, espressamente destinate alla manutenzione di santuari riservati al culto reale. Ulteriore impulso alla celebrazione del culto reale fu conferito dalle largizioni evergetiche, rese in misura prevalente dagli ufficiali dell'esercito tolemaico. Un sommario confronto fra la documentazione tolemaica e le testimonianze legate al culto reale dei Seleucidi non mostra, nel caso di questi ultimi, un impegno altrettanto costante e sollecito nella promozione e nella diffusione dei rituali in onore dei membri della propria dinastia.

9 Frutto della collaborazione fra Caneva e Lorenzon è l'ultimo contributo del volume, incentrato sugli inni composti in onore di sovrani e di altre personalità politiche di primo piano («Les hymnes pour les chefs politiques dans les fêtes civiques: l'apport local à la construction des mythologies royales», pp. 195-226). Caneva, da un lato, si sofferma dapprima sulle procedure di selezione dei poeti e delle loro opere da parte 
delle comunità cittadine, individuando in seguito i momenti rituali nei quali i componimenti venivano pubblicamente declamati. Metodologicamente rilevanti sono le riflessioni a proposito dei contenuti sviluppati all'interno di tali inni, i quali dovevano rispondere non soltanto alle esigenze del corpo civico di celebrare un suo (potenziale) benefattore, ma dovevano intercettare anche le principali istanze della propaganda reale. Lorenzon, d'altro canto, indaga un celebre caso di studio come la discendenza apollinea di Seleuco Nicatore, di cui si ha notizia più antica nell'incipit del peana per il sovrano, inciso su una stele da Eritre: l'esempio ben illustra in che modo l'elaborazione di un nesso genetico fittizio fra Seleuco e Apollo, autorevolmente avallato dall'oracolo didimeo, giunga a combinare le ambizioni di legittimazione della dinastia seleucide e l'esigenza di ottenere prestigio e risorse da parte del santuario milesio.

10 Chiudono il volume una nota conclusiva a cura di Caneva («Afterword», pp. 227-239), nella quale si ripercorrono ordinatamente i risultati e gli elementi di novità scaturiti dalla pubblicazione, l'elenco dei contributori (pp. 241-242) e i rispettivi abstracts (pp. 243-248), la bibliografia (pp. 249-281) e infine gli indici (pp. 282-299).

11 L'ambizione del volume, in conclusione, è certamente quella di risvegliare l'attenzione sugli aspetti materiali del culto reale, mettendo in luce il potenziale euristico insito in un loro accurato esame. Sotto questo profilo, non v'è dubbio che i propositi iniziali si siano trasformati effettivamente nel principale pregio di questa ricca miscellanea, i cui contributi - seguendo l'approccio pluridisciplinare indicato in sede d'introduzione toccano aspetti archeologici e questioni epigrafiche; ridiscutono problemi di iconografia e riconsiderano sotto una diversa luce la documentazione papiracea; valorizzano l'evidenza numismatica e recuperano nelle fonti letterarie informazioni essenziali (talvolta trascurate) sull'organizzazione del culto reale. È tuttavia da chiedersi se il pur ambizioso tentativo di declinare la materiality in numerose delle sue possibili sfaccettature non rischi di segnare il limite di una simile intrapresa, la quale in ultima analisi - consegna ai fruitori del volume una mole di dati non del tutto omogenei, quasi rimettendo loro l'onere di una non semplice sintesi. Sotto questo rispetto, la Afterword compensa almeno in parte questo senso di smarrimento, dando occasione al Curatore di ripercorrere pazientemente gli itinerari di ricerca proposti e facendone così riaffiorare i principali traits-d'-union tematici.

\section{AUTORI}

\section{CLAUDIO BIAGETTI}

Westfälische Wilhelms-Universität Münster

Seminar für Alte Geschichte / Institut für Epigraphik

Domplatz 20-22

48143 - Münster, Germania

claudio.biagetti(at)hotmail.it 